Service social

\title{
Les représentations sociales des droits de l'homme et les valeurs chez des étudiants francophones à Québec
}

\section{Monica Herrera et Marguerite Lavallée}

Volume 45, numéro 2, 1996

Droit et pratiques sociales

URI : https://id.erudit.org/iderudit/706728ar

DOI : https://doi.org/10.7202/706728ar

Aller au sommaire du numéro

\section{Éditeur(s)}

École de service social de l'Université Laval

ISSN

1708-1734 (numérique)

Découvrir la revue

Citer cet article

Herrera, M. \& Lavallée, M. (1996). Les représentations sociales des droits de l'homme et les valeurs chez des étudiants francophones à Québec. Service social, 45(2), 101-128. https://doi.org/10.7202/706728ar
Résumé de l'article

La présente étude, menée auprès d'étudiantes et d'étudiants francophones québécois, analyse les représentations sociales des droits de l'homme (RSDH). Par le moyen d'un questionnaire basé sur la Déclaration universelle des droits de l'homme (1948), cette recherche examine les structures du champ des RSDH et l'ancrage de ces champs dans le système des valeurs et par rapport aux perceptions de tensions et d'injustices personnelles liées à différentes catégories sociales. Les résultats montrent que même si les sujets partagent une structure commune du champ des RSDH semblable aux classifications institutionnelles, et plus spécifiquement à celle de René Cassin, des variations interindividuelles apparaissent également dans le champ de ces

représentations. En outre, à partir des évaluations demandées au sujet des 30 articles de la Déclaration, deux principes organisateurs (contributions gouvernementales / contributions personnelles) sont mis en évidence. À partir de ces principes, trois profils d'individus sont dégagés: les optimistes, les personnalistes et les gouvernementalistes. Ils se distinguent par les différents ancrages dans leur structure de valeurs, leurs perceptions de tensions entre diverses catégories sociales et d'injustices sociales personnelles ressenties à leur égard. 


\title{
CONTRIBUTION INTERNATIONALE
}

\section{Les représentations sociales des droits de l'homme et les valeurs chez des étudiants francophones à Québec ${ }^{1}$}

\author{
Monica HERRERA \\ Assistante de recherche \\ Laboratoire de psychologie sociale \\ Université de Genève, Suisse \\ Marguerite LAVALLÉE \\ Professeure titulaire \\ École de psychologie, Université Laval
}

Ce monument, inspiré par un idéalisme pratique, repose sur l'unité de la famille humaine et fournit, malgré ses inévitables imperfections, la base d'une éthique sans laquelle la société universelle ne pourrait s'organiser sur les plans moral, politique, juridique et même économique...

René Cassin, La Déclaration universelle et la mise en auvre des droits de l'homme. 


\section{INTRODUCTION}

Un peu partout dans le monde, à la suite des transformations profondes que connaissent nos sociétés contemporaines, de nouveaux rapports sociopolitiques et économiques se dessinent à l'échelle internationale. La gestion des échanges entre membres de sociétés différentes est inscrite à l'ordre du jour. L'enjeu est de taille, puisqu'il s'agit de rapports qui affectent les racines mêmes des cultures en contact, chacune dotée de codes et de valeurs qui sont propres à son identité. Constituée d'un ensemble de sous-groupes (ethniques, régionaux, de classes) plus ou moins différenciés, chaque société fait face au souci de faire respecter les valeurs dont ils se sont dotés et de réagir devant toute distribution inégale des droits et du pouvoir (Passeron, 1991).

On connaît par ailleurs les effets pervers que peuvent engendrer les actions sociales qui, trop centrées sur les différences culturelles, font fi des contextes où elles s'expriment et des interactions qu'elles entretiennent avec les autres réalités sociales (Boudon, 1977: 1995). Aussi est-on en droit de se demander si, au-delà des diversités qu'il est légitime de vouloir protéger, il n'existe pas des espaces communs qui unissent les individus entre eux et leur permettent de se rencontrer dans le respect des uns et des autres?

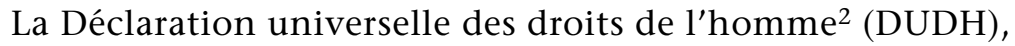
promulguée en 1948, vise à constituer cette base commune nécessaire à toute société. Ainsi a-t-elle été érigée en norme générale de justice sociale qui transcende toute frontière, pour rejoindre les droits légitimes et les devoirs de tout citoyen. L'idée d'un code moral universel n'est pas nouvelle; elle s'est imposée à des moments historiques cruciaux (Révolution française, Seconde Guerre mondiale) où justement les droits fondamentaux des personnes étaient bafoués au profit d'enjeux politiques et économiques. À la base, ces déclarations reposent sur deux principes étroitement liés dont les composantes ne sont pas facilement réconciliables: l'unité de l'espèce humaine au-delà des diversités des ethnies, groupes et individus et l'universalité des valeurs humaines fondamentales au-delà des relativités culturelles (voir Gauchet, 1989; Bercis, 1993). La reconnaissance de ces droits tire donc sa signification de dynamiques relationnelles complexes. En outre, l'élaboration formelle d'un tel code ne s'est pas faite sans problème: c'est par un jeu de compromis entre défenseurs de libertés individuelles et partisans de droits sociaux et économiques régis par l'État que la dernière Charte a finalement pu être acceptée. 
À la veille du $50^{\mathrm{e}}$ anniversaire de cette déclaration, les droits qu'elle promulgue sont devenus des référents incontournables pour régler les nombreux conflits et tensions nationaux et internationaux qui existent à différents points du globe et les nombreuses revendications qui émergent pour en exiger le respect au nom d'intérêts et d'attentes variables. Cependant, ces mêmes conflits et tensions révèlent que les conceptions des droits de l'homme (DH) parmi les nations, les peuples et les individus, même si elles sont partagées, ne sont pas forcément consensuelles. À cet effet, Bourdieu (1991) souligne que tout droit et la dynamique de son application doivent être envisagés en tant que produit des processus transhistorique et transsocial. De ce fait, et sans que soient pour autant anéanties leurs prétentions à l'universalité, les DH doivent être abordés en tenant compte des contextes et événements spécifiques relatifs aux acteurs sociaux (États, nations, individus) qui les actualisent.

La théorie des représentations sociales (RS), domaine récemment développé en psychologie sociale, est une voie prometteuse pour mieux comprendre ces processus. Moscovici (1976) a démontré comment toute représentation sociale peut être considérée comme une modalité de connaissance ayant pour fonction d'orienter les comportements et de permettre la communication entre individus. Dans cette perspective, l'étude des représentations sociales des droits de l'homme (RSDH) permet d'aborder la problématique de l'universalité de ces droits à partir de l'analyse du champ des savoirs communs, partagés par des ensembles sociaux, et de l'ancrage de ces savoirs dans le monde symbolique des individus et dans leurs diverses insertions, soient-elles sociale, culturelle ou nationale.

Les différents travaux sur les RSDH, menés ces dernières années, vont dans ce sens. Ils mettent en évidence, entre autres, des aspects universels et certaines particularités dans les conceptions novices des DH. Ainsi a-t-on observé auprès de diverses populations une connaissance commune de certains droits dits fondamentaux (p. ex. égalité, justice, liberté), de même que des interdits de violation qui s'y rattachent (voir Doise, Dell'Ambrogio et Spini, 1991). Par ailleurs, une étude menée à Genève (Doise et Herrera, 1994) révèle une vision des DH qui transcende la conception juridique formelle; les sujets interrogés semblent privilégier les $\mathrm{DH}$ en fonction des intérêts propres à leurs groupes d'appartenance socioprofessionnelle. Une autre étude menée auprès de populations de quatre pays 
(Costa Rica, France, Italie et Suisse) a montré : a) la présence de principes organisateurs du champ des RSDH communs aux divers groupes et $b$ ) des variations interindividuelles et intergroupes liées aux insertions sociales et nationales des individus concernés (Clémence, Doise, De Rosa et Gonzales, 1995). L'équipe de Genève, sous la direction de W. Doise, a entrepris une étude plus vaste des RSDH dans plus de 30 pays des cinq continents. Elle vise à étudier l'universalité des représentations des DH tant sur le plan de leur contenu que de l'organisation de ce contenu. Les chercheurs font l'hypothèse qu'il existe un savoir commun des DH chez les populations étudiantes de ces différents pays. Ils cherchent également à vérifier si ce savoir s'organise selon une structure semblable à celle proposée par Cassin ${ }^{3}$. Enfin, ils étudient les liens qu'ont ces représentations avec certaines variables psychosociales, tels les valeurs, le degré de sensibilisation aux conflits et tensions entre différentes catégories de groupes sociaux (âge, sexe, race, etc.). Les résultats des données récoltées dans trois pays (Nouvelle-Zélande, Portugal et Suisse) appuient ces hypothèses (Doise, Spini, Jesuino, Ng et Emler, 1994).

La présente étude, menée en milieu québécois, s'inscrit dans ce projet transculturel. Cette société apparaît intéressante pour étudier les RSDH. En effet, composée d'une population en majorité francophone dont elle tire son principal trait distinctif, elle a, durant toute son histoire, été confrontée à la réalité linguistique anglophone, minoritaire au Québec mais très majoritaire au Canada et en Amérique du Nord en général. Si l'on ajoute à cette configuration la présence de communautés autochtones et de divers groupes ethniques et allophones, la province de Québec revêt un caractère multiethnique indéniable. Cette situation n'est pas sans créer des tensions et conflits entre les différents partenaires en présence qui, au nom des droits de la personne, revendiquent que soit respecté leur héritage culturel respectif (Gay, 1985 ; Côté et Lemonde, 1988; Yalden, 1988).

Le Québec a par ailleurs connu des transformations très importantes, surtout depuis la Révolution tranquille des années 1960. D'une société qui se caractérisait par une organisation familiale fondée sur l'attachement à la terre, l'esprit d'indépendance et le primat des relations personnelles dans la conduite des affaires, de même que par l'omniprésence de la religion catholique, elle est passée à une définition identitaire moins traditionnelle et défensive, orientée vers une appropriation du pouvoir économique et vers une amélioration du statut socioéconomique de ses membres (Rocher et Rocher, 1991). Le climat 
d'incertitude devant l'avenir qui prévaut depuis quelques années au Québec est alimenté par ces changements qui modifient progressivement sa structure sociale et culturelle: décroissance démographique très marquée, urbanisation accrue et dépeuplement des zones rurales, venue d'immigrants de plus en plus «visibles», concentration urbaine des communautés ethniques, sentiment d'être menacé linguistiquement, autant de paramètres qui illustrent le poids des enjeux et laissent présager une dynamique relationnelle très complexe. Ce n'est pas par hasard que le Québec, conscient des défis qu'il a à relever pour sauvegarder ses acquis politiques et culturels, s'est doté dans les années 1970 d'une véritable politique d'immigration et a récupéré du gouvernement fédéral des pouvoirs directement ou indirectement liés à ces enjeux.

Dans ce contexte particulier, il semblait intéressant de se pencher sur certains aspects relatifs au savoir commun des Québécois sur les DH pour ensuite dégager les relations qu'entretient ce savoir commun, d'une part, avec certaines valeurs jugées importantes par les Québécois et, d'autre part, avec les tensions qu'ils perçoivent entre différentes catégories de groupes sociaux et avec certaines injustices dont ils ont été eux-mêmes l'objet. En fait, deux objectifs sont poursuivis dans la présente recherche. Il s'agit a) de mettre en évidence les structures et certaines dimensions significatives du champ des RSDH et b) d'examiner l'ancrage de ces représentations dans les systèmes de valeurs jugés importants par les sujets et par rapport aux tensions qu'ils disent percevoir entre certaines catégories sociales et aux injustices qu'ils disent avoir personnellement vécues.

\section{MÉTHODE}

\section{La population}

La population est composée d'étudiants et d'étudiantes francophones $(n=488)$ du Québec. Pour une première collecte de données (janvier 1995), ont participé 286 étudiants $(58,6 \%)$. La plupart sont inscrits à l'Université Laval à Québec $(\mathrm{n}=241)$; les autres proviennent des deux universités francophones de Montréal $^{4}(n=45)$. Leurs champs d'études sont le droit $(n=71)$, les sciences $(n=101)$, le travail social $(n=100)$, la psychologie $(n=15)$. Pour la deuxième collecte (octobre 1996), ont participé 202 étudiants $(41,4 \%)$, dont la plupart sont de l'Université Laval 
$(\mathrm{n}=136)$ et les autres du collège François-Xavier-Garneau à Québec $(n=66)$. Leurs champs d'études sont la science politique et la sociologie ${ }^{5}(n=105)$, la psychologie $(n=30)$ et, chez les collégiens, les sciences humaines $(n=66)$. Le tableau 1 présente le sexe, les moyennes d'âge et les pourcentages des personnes répondantes qui ont participé aux deux études $(n=488)$.

\section{Tableau 1}

Sexe, moyenne d'âge et pourcentage des participants aux deux études

\begin{tabular}{lrrccc}
\hline Groupes d'étudiants & $\mathbf{n}$ & \% & Âge moyen & \% hommes & \% femmes \\
\hline Sciences humaines (collège) & 66 & 13,5 & 20,2 & 43,9 & 56,1 \\
Droit & 71 & 14,5 & 28,5 & 32,4 & 66,2 \\
Psychologie & 45 & 9,0 & 22,9 & 26,7 & 68,9 \\
$\begin{array}{l}\text { Sciences } \\
\text { Science politique }\end{array}$ & 101 & 21,0 & 24,1 & 57,4 & 42,6 \\
et sociologie & 105 & 21,5 & 24,7 & 53,3 & 46,7 \\
Travail social & 100 & 20,5 & 28,7 & 21,0 & 79,0 \\
\hline Total & 488 & $\mathbf{1 0 0 , 0}$ & $\mathbf{2 5 , 2}$ & $\mathbf{4 0 , 8}$ & $\mathbf{5 8 , 6}$ \\
\hline
\end{tabular}

\section{Questionnaire}

La première partie des deux collectes de données est réalisée à partir du questionnaire de Doise et al. (1994), utilisé pour l'étude transculturelle des RSDH dans plus de 30 pays. Ce questionnaire comporte deux parties:

- La première partie contient les 30 articles de la DUDH (1948). Les sujets sont invités à se prononcer sur chaque article à partir des huit échelles suivantes :

1) «article difficile (facile) à comprendre»;

2) «mesures difficiles (faciles) à appliquer par les gouvernements »;

3) «implications claires (pas d'implications claires) pour la responsabilité des individus envers les autres »;

4) «les partis politiques peuvent faire (ne peuvent pas faire) beaucoup pour défendre cet article»;

$5)$ «le contenu de cet article est pertinent (n'est pas pertinent) pour ma vie privée»; 
6) «je peux (je ne peux pas) faire beaucoup pour le respect de cet article»;

7) « je suis (je ne suis pas) d'accord avec tous les aspects de cet article tel qu'il est rédigé» ;

8 ) «je suis prêt(e) (je ne suis pas prêt[e]) à joindre mes efforts pour faire respecter les différents aspects de cet article à ceux d'autres personnes concernées ».

- La deuxième partie comporte plusieurs sections; sont décrites ici celles qui se rapportent à la présente étude.

1. Valeurs. Cette échelle est composée des 18 valeurs instrumentales de l'échelle de Rokeach (1973), de deux items de Bond (1988): " harmonie avec les autres (sociable, accommodant) et respect des traditions (perpétuation des rites et des coutumes) » et de 11 items tirés du Préambule de la Déclaration universelle des droits de l'homme (1948), (p. ex.: «dignité pour tous les humains, liberté partout dans le monde, absence de terreur et de misère, etc. »). L'importance accordée à chaque item est évaluée à l'aide d'une échelle Likert à neuf positions $(1=$ pas du tout important à $9=$ extrêmement important), selon la procédure utilisée par $\mathrm{Ng}$ et al. (1982) et Doise et al. (1994).

2. Perception de tensions et de conflits selon les différences sociales entre individus. Les catégories considérées dans cette question sont tirées du deuxième article de la charte des DH (p. ex. : race, sexe, langue, origine sociale, etc.). La question posée est «Dans quelle mesure y a-t-il des tensions ou des conflits entre individus... de races différentes, de sexes différents, de langues différentes...?». Les répondants se prononcent sur une échelle de quatre points $(1=\mathrm{il}$ y a très peu de tensions et de conflits à $4=\mathrm{il} \mathrm{y}$ a beaucoup de tensions et de conflits).

3. Perception d'injustices personnelles. Les items de cette section se rapportent aussi au deuxième article de la DUDH. La question posée est «Dans quelle mesure, si cela vous est arrivé, avez-vous été personnellement traité de manière injuste à cause des raisons suivantes...? ». Les sujets se prononcent sur une échelle de quatre points $(1=$ jamais à $4=$ très fréquemment). 
4. La dernière section regroupe des questions sociodémographiques (sexe, statut civil, etc.).

La première partie du questionnaire a été administrée à l'ensemble de la population $(\mathrm{n}=488)$, la deuxième seulement aux répondants de la première collecte des données $(\mathrm{n}=286)$.

\section{RÉSULTATS}

\section{L'analyse des données}

Les analyses effectuées s'inspirent d'une nouvelle approche théorique et méthodologique des RS, proposée par Doise, Clémence et Lorenzi-Cioldi (1992), et d'un premier article portant sur l'étude transculturelle des DH (Doise et al., 1994).

L'évaluation des 30 articles des DH permet d'analyser l'objectivation, c'est-à-dire le savoir commun à l'égard des DH. Plus spécifiquement, à travers les huit échelles considérées dans la première partie du questionnaire, on cherche à dégager la structure et les principes organisateurs des différences individuelles du champ des RSDH. Les analyses de cette partie sont effectuées à partir des réponses de l'ensemble de la population $(n=488)$. Les analyses de la deuxième partie du questionnaire permettent d'étudier l'ancrage par rapport aux valeurs et à la perception de tensions selon certaines catégories de groupes sociaux et d'injustices personnelles en lien avec ces mêmes catégories. Ces analyses ne portent que sur les données recueillies auprès des sujets du premier échantillon $(\mathrm{n}=286)$.

\section{Le savoir commun}

Des analyses multidimensionnelles (MDS) sont d'abord utilisées pour dégager la structure du champ commun des RSDH. La procédure d'analyse est la suivante:

1. On obtient des coefficients de dissimilitude entre chaque paire d'objets (30 articles) selon les huit échelles ${ }^{6}$.

2. À partir de ces coefficients, une analyse d'agrégation est réalisée pour obtenir des matrices de distance. Pour contrôler la variabilité interindividuelle, le nombre de matrices a été limité à six (représentant les six champs d'études des sujets (collégiens en sciences humaines, étudiants en droit, psychologie, sciences, science politique-sociologie et travail social). Chaque matrice a été construite à partir des moyennes des coefficients de chaque groupe de sujets. 
3. Une analyse INDSCAL (INdividual Differences multidimensional SCALing) est ensuite effectuée sur ces six matrices. De cette analyse, on a retenu une solution à trois dimensions (voir tableau 2) qui se révèle consistante par son haut pourcentage de variance expliquée $(76 \%)$ et une valeur conventionnelle de stress $(0,199)$. L'importance des trois dimensions est semblable $(0,29,0,24$ et 0,22$)$.

TABLEAU 2

Valeurs des articles des DH sur les trois dimensions obtenues

\begin{tabular}{|c|c|c|c|c|}
\hline \multirow[b]{2}{*}{ Items } & \multirow[b]{2}{*}{ Articles } & \multicolumn{3}{|c|}{ Dimensions } \\
\hline & & 1 & 2 & 3 \\
\hline 1 & Égalité & $-2,76$ & 0,09 & 0,15 \\
\hline 2 & Non-discrimination & $-2,57$ & 1,64 & $-0,35$ \\
\hline 3 & Droit à la vie & $-1,66$ & $-0,62$ & $-0,75$ \\
\hline 4 & Interdiction d'esclavage & $-0,90$ & $-1,27$ & $-1,97$ \\
\hline 5 & Interdiction de torture & $-1,33$ & $-0,99$ & $-1,07$ \\
\hline 6 & Reconnaissance juridique & 0,63 & 2,81 & $-1,31$ \\
\hline 7 & Égalité devant la loi & $-0,26$ & 0,77 & $-0,87$ \\
\hline 8 & Recours juridique & 0,56 & 1,45 & $-0,97$ \\
\hline 9 & Non-arrestation arbitraire & 0,42 & $-0,12$ & $-1,61$ \\
\hline 10 & Équité juridique & 0,49 & 0,11 & $-0,95$ \\
\hline $11 \mathrm{~J}$ & Jugement équitable & 1,35 & 0,50 & $-1,01$ \\
\hline 12 & Vie privée & $-0,47$ & 0,86 & 1,51 \\
\hline 13 & Libre circulation & 0,70 & $-0,98$ & $-0,77$ \\
\hline 14 & Asile & 1,42 & 0,69 & $-0,61$ \\
\hline 15 & Nationalité & 1,29 & $-0,74$ & $-0,75$ \\
\hline 16 & Mariage & 0,08 & $-1,61$ & 0,62 \\
\hline 17 & Propriété & 0,37 & $-0,88$ & $-0,04$ \\
\hline 18 & Liberté de pensée & $-0,27$ & $-0,88$ & 1,82 \\
\hline 19 & Liberté d'opinion & $-0,02$ & $-0,58$ & 1,28 \\
\hline 20 & Libre association & $-0,03$ & $-0,77$ & 0,23 \\
\hline 21 & Démocratie & 0,82 & $-0,59$ & $-0,12$ \\
\hline 22 & Sécurité sociale & 0,79 & 0,51 & 1,20 \\
\hline 23 & Travail & $-0,39$ & $-0,61$ & 0,83 \\
\hline 24 & Repos & $-0,19$ & $-0,99$ & 0,43 \\
\hline 25 & Niveau de vie suffisant & $-0,17$ & $-0,33$ & 0,35 \\
\hline 26 & Éducation & $-0,06$ & $-0,55$ & 0,87 \\
\hline 27 & Vie culturelle & 0,20 & 0,12 & 0,80 \\
\hline 28 & Ordre public & 0,66 & 0,63 & 1,01 \\
\hline 29 & Devoirs envers la communauté & 0,61 & 1,48 & 1,28 \\
\hline 30 & Ordre international & 0,68 & 0,84 & 0,77 \\
\hline
\end{tabular}


Dans la première dimension, les articles (1 à 5) dits fondamentaux (égalité, non-discrimination, droit à la vie, interdiction d'esclavage, interdiction de torture) reçoivent les valeurs négatives les plus extrêmes par rapport aux autres droits. Quant aux articles 6 (droit à la reconnaissance juridique), 11 (droit au jugement équitable), 13 (droit à la libre circulation), 14 (droit à l'asile), 15 (droit d'avoir une nationalité), 21 (droit à la démocratie), 22 (droit à la sécurité sociale), 28 (droit à l'ordre public), 29 (devoirs à l'égard de la communauté), 30 (droit à l'ordre international), ils obtiennent les valeurs positives les plus élevées. Cette dimension montre une opposition entre les droits dits fondamentaux et les droits qui touchent l'immigration, les devoirs et l'ordre national et international; elle permet d'inférer une relation inverse entre les droits fondamentaux et ceux qui stipulent la sécurité sociale, la démocratie, l'ordre social et qui sollicitent la responsabilité des individus à l'égard de leur communauté. Cette dimension illustre ainsi la double composition de la DUDH, à savoir les droits et les devoirs de tout individu.

Dans la deuxième dimension, les articles dont les valeurs négatives sont les plus élevées sont les suivants : 3 (droit à la vie), 4 (interdiction d'esclavage), 5 (interdiction de torture), 13 (libre circulation), 15 (nationalité), 16 (mariage), 17 (propriété), 18 (liberté de pensée), 20 (libre association), 23 (travail), 24 (repos). Les articles aux valeurs positives les plus élevées sont: 2 (non-discrimination), 6 (reconnaissance juridique), 7 (égalité devant la loi), 8 (recours juridique), 12 (vie privée), 14 (asile), 28 (ordre public), 29 (devoirs envers la communauté) et 30 (ordre international).

Cette dimension met en évidence une polarisation entre la notion de libre circulation associée aux causes d'immigration (torture, esclavage, travail, mariage, propriété, association, liberté de pensée) et la notion d'asile liée à la non-discrimination, à la reconnaissance juridique, à la vie privée, aux devoirs envers la communauté et à l'ordre international. Cette opposition indique que le premier pôle (libre circulation) est lié à des droits socioéconomiques, alors que le deuxième (asile) est plutôt associé au droit fondamental de non-discrimination et à des droits civils et politiques. Le lien qui se dégage entre droit à l'asile, devoirs envers la communauté et ordre international permet d'inférer une dynamique régulatrice de ces droits par rapport au statut de réfugié.

Dans la troisième dimension, les articles 3 à 11 (droits de la personne), 13 à 15 (droits des rapports entre les personnes) 
affichent les valeurs négatives les plus élevées par opposition aux articles 18 et 19 (droits des libertés publiques), 22, 23, 26 et 27 (droits économiques et sociaux), 28 à 30 (droits à l'ordre international) qui montrent les valeurs positives les plus élevées. Cette dimension révèle une polarisation entre les droits qui touchent la sphère individuelle (droits de la personne et des rapports entre les personnes) et ceux qui touchent la sphère communautaire (droits économiques et sociaux, libertés publiques, devoirs envers la communauté, ordre international).

L'examen des poids des six groupes d'individus sur ces trois dimensions offre la configuration suivante. L'utilisation la plus fréquente de la première dimension, droits fondamentaux versus responsabilités des individus à l'égard de la communauté, est faite par les répondants de science politique-sociologie, de sciences et par les collégiens, suivis des groupes de travail social et de droit. Le groupe de psychologie est celui qui obtient le poids le moins élevé sur cette dimension. Pour la deuxième dimension, libre circulation versus asile, le poids le plus élevé est observé chez le groupe de travail social, qui se distingue nettement des autres groupes. Pour sa part, le groupe de psychologie obtient un poids modéré, suivi de près des groupes des collégiens, de sciences et de science politique-sociologie. Le groupe de droit est celui qui l'utilise le moins. En ce qui a trait à la troisième dimension, sphère individuelle versus sphère communautaire, ce sont les groupes de droit et de psychologie qui l'utilisent le plus, suivis de près par les groupes de science politique-sociologie, de sciences et les collégiens. Le groupe de travail social est celui qui l'utilise le moins.

Ainsi, les groupes de science politique-sociologie, de sciences et les collégiens affichent un pattern semblable pour les trois dimensions: la première prime sur les deux autres, qui sont utilisées modérément. Les patterns des trois groupes restants sont plus nuancés et se différencient les uns des autres. Le groupe de travail social utilise les deux premières dimensions, mais surtout la deuxième; il accorde peu d'importance à la troisième dimension. Quant au groupe de psychologie, il se montre plus saillant sur les deux dernières dimensions et peu sur la première. Enfin, le groupe de droit privilégie la troisième dimension, utilise modérément la première et n'attache que peu d'importance à la deuxième.

4. Les cœfficients obtenus pour les 30 articles sur les trois dimensions mettent en évidence les similarités à travers 
une analyse de classification hiérarchique. Les indices de similarité sont obtenus par la méthode de Ward qui permet une classification plus homogène des objets (articles).

L'analyse de classification hiérarchique montre quatre classes de droits : 1) la classe des droits élémentaire, qui regroupe les articles 1 à 5 ; selon la classification de Cassin, les articles 1 et 2 sont des droits fondamentaux; les articles 3 à 5 appartiennent à la catégorie de la personne; 2 ) la classe des droits civils et politiques, constituée des articles relatifs aux statuts juridiques des individus; selon Cassin, les articles 6 à 11 correspondent aux droits de la personne et l'article 14 aux rapports entre les personnes; 3 ) la classe des droits socio-économiques, qui contient les articles 13,15 à 21 et 23 à 26 ; selon Cassin, les articles 13 et 15 à 17 sont des droits de rapports entre les personnes, les articles 18 à 21 énoncent des libertés publiques et les articles 23 à 26 sont des droits économiques, sociaux et culturels ; 4) la classe des droits de l'ordre social, composée des articles 12, 22 et 27 à 30 ; selon Cassin, l'article 12 appartient aux droits des rapports entre les personnes, les articles 22 et 27 aux droits socio-économiques et les articles 28 à 30 aux droits relatifs à l'ordre international.

Ainsi, la structure générale dégagée par la classification hiérarchique montre une organisation des droits semblable à la classification proposée par Cassin. Les résultats de ce classement rejoignent également ceux déjà obtenus dans d'autres travaux (Doise et Herrera, 1994 ; Doise et al., 1994).

\section{Les principes organisateurs des différences interindividuelles}

Pour dégager les principes organisateurs des différences interindividuelles, une analyse factorielle en composantes principales a été effectuée sur un indice ${ }^{7}$ calculé à partir de la moyenne des réponses sur les 30 articles pour chacune des huit échelles; deux facteurs expliquant $60 \%$ de la variance sont obtenus. Cependant, l'échelle 2 qui évalue le rôle des partis politiques («les partis politiques peuvent faire/ne peuvent pas faire beaucoup pour défendre cet article») et l'échelle 3 qui évalue la responsabilité des individus ( a des implications/n'a pas d'implications claires pour la responsabilité des individus envers les autres») étant fortement saturées sur les deux facteurs, une deuxième analyse est effectuée sur les six autres échelles. De cette deuxième analyse, les deux facteurs obtenus expliquent $67 \%$ de la variance; le facteur 1 en explique $50 \%$ et le facteur 2, 17\% (tableau 3). 
Figure 1

Représentation graphique de l'analyse de classification hiérarchique (méthode de Ward) des distances entre les 30 articles de la DUDH en tenant compte des trois dimensions obtenues par I'analyse INDSCAL avec indication des classes de Cassin

\begin{tabular}{|c|c|c|c|c|c|}
\hline Articles & & Dis & aclic & & \\
\hline 0 & 5 & 10 & 15 & 20 & 25 \\
\hline
\end{tabular}

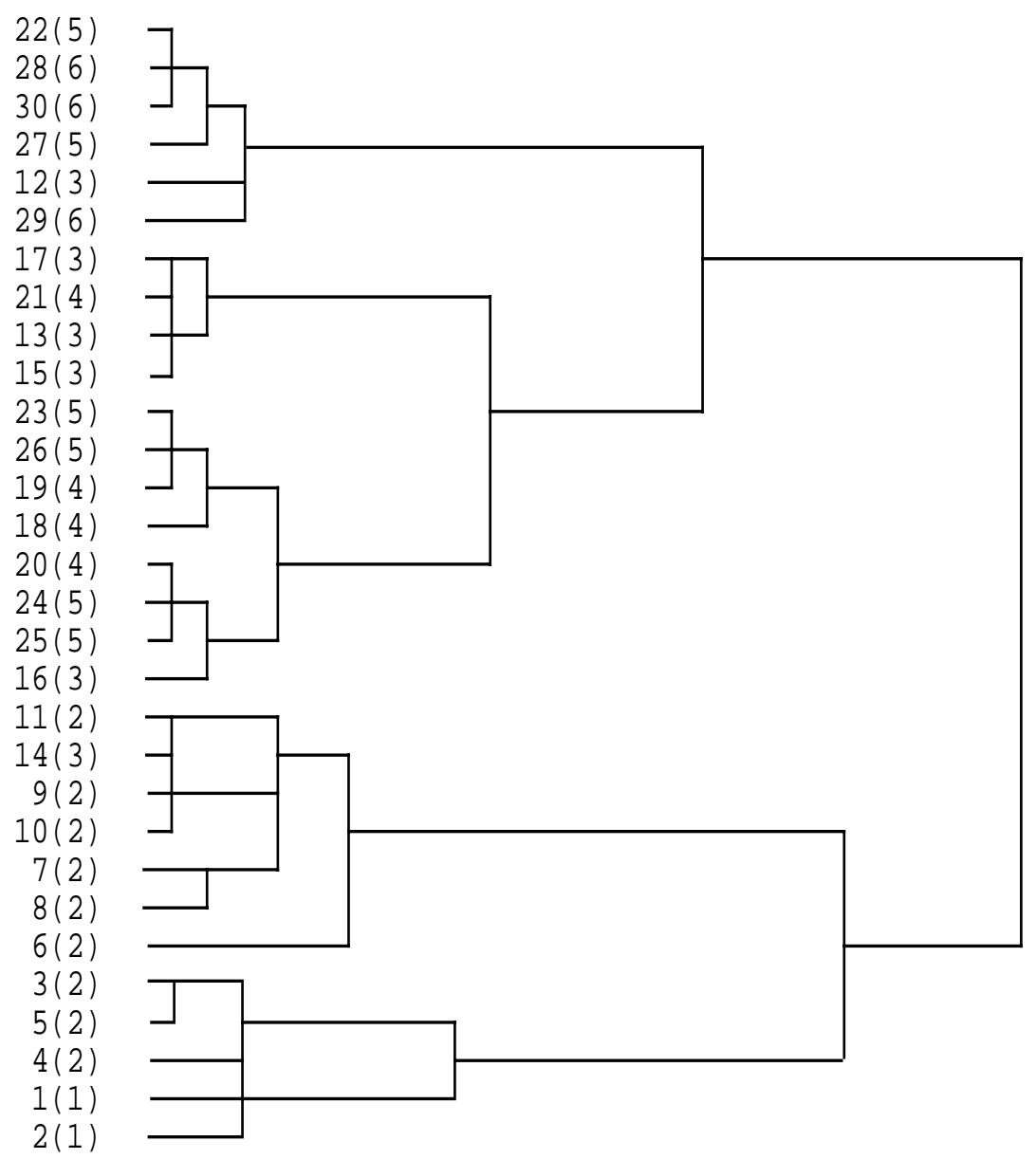

Note: Les nombres entre parenthèses correspondent aux six classes de droits selon Cassin dans l'ordre DUDH ( 1 = fondamentaux, 2 = personnels, $3=$ relationnels, 4 publics, $5=$ économiques et $6=d^{\prime}$ ordre international). 
Tableau 3

Résultats de l'analyse factorielle des six échelles d'évaluation des $\mathrm{DH}$ retenues

\begin{tabular}{lccc}
\hline \multirow{2}{*}{ Échelles } & \multicolumn{2}{c}{ Saturations } & \\
\hline 6) Contribution individuelle & $\mathbf{0 , 8 3}$ & 0,15 & Communauté \\
5) Importance pour la vie privée & $\mathbf{0 , 7 9}$ & 0,20 & 0,71 \\
8) Efforts mis en commun & $\mathbf{0 , 7 9}$ & 0,24 & 0,67 \\
1) Compréhension & 0,04 & $\mathbf{0 , 8 0}$ & 0,68 \\
2) Rôle du gouvernement & 0,27 & $\mathbf{0 , 7 8}$ & 0,68 \\
7) Accord & 0,36 & $\mathbf{0 , 6 9}$ & 0,64 \\
Pourcentage de variance expliquée: & $50 \%$ & $17 \%$ & 0,60 \\
\hline
\end{tabular}

Note: Saturations et communauté après rotation varimax.

De cette analyse, deux principes organisateurs ressortent clairement: a) un principe organisateur de contribution et effort personnels liés à l'importance des articles pour la vie privée et b) un deuxième, de contribution gouvernementale en relation avec l'accord et la compréhension des droits.

3. Regroupement des individus selon les principes organisateurs et les classes de DH obtenus

Une analyse de classification non hiérarchique (K-means) a été effectuée pour distinguer les répondants de la première collecte de données $(n=286)$, en fonction des deux principes organisateurs et des quatre classes de droits dégagées. Cette analyse a été effectuée en utilisant la procédure Quick Cluster de SPSS, appliquée sur un indice construit à partir des moyennes de réponses des principes organisateurs dégagés dans chacune des quatre classes de droits obtenues par l'analyse de classification hiérarchique ( 2 principes organisateurs $\times 4$ classes de droits). Une solution à trois groupes a été retenue, un premier groupe avec 68 sujets $(23,8 \%)$, un deuxième avec $146(51 \%)$ et un troisième avec $72(25,2 \%)$. Le tableau 4 indique les moyennes des réponses obtenues pour chaque groupe sur les deux principes organisateurs et les quatre classes de droits. 


\section{TABleau 4}

Moyennes des réponses des trois groupes obtenues par Q-analyse selon les deux principes organisateurs et les quatre classes de droits

\begin{tabular}{|c|c|c|c|c|c|}
\hline \multirow[b]{2}{*}{ Principes organisateurs } & \multicolumn{5}{|c|}{ Classes de droits selon la dassification obtenue } \\
\hline & & $\begin{array}{c}\text { Élémen- } \\
\text { taires }\end{array}$ & $\begin{array}{c}\text { Civils- } \\
\text { politiques }\end{array}$ & $\begin{array}{l}\text { Socio- } \\
\text { économ. }\end{array}$ & $\begin{array}{l}\text { Ordre } \\
\text { social }\end{array}$ \\
\hline \multicolumn{6}{|l|}{ Contributions personnelles } \\
\hline \multicolumn{6}{|l|}{ Groupes } \\
\hline 1. Personnaliste & $(\mathrm{n}=68)$ & 7,40 & 6,66 & 7,07 & 6,66 \\
\hline 2. Optimiste & $(n=146)$ & 7,72 & 7,23 & 7,71 & 7,42 \\
\hline 3. Gouvernementaliste & $(n=72)$ & 5,70 & 4,66 & 5,65 & 5,06 \\
\hline \multicolumn{6}{|c|}{ Contributions gouvernementales } \\
\hline \multicolumn{6}{|l|}{ Groupes } \\
\hline 1. Personnaliste & $(\mathrm{n}=68)$ & 6,55 & 6,23 & 6,63 & 6,01 \\
\hline 2. Optimiste & $(n=146)$ & 8,09 & 7,95 & 8,15 & 7,79 \\
\hline 3. Gouvernementaliste & $(n=72)$ & 7,02 & 6,58 & 6,88 & 5,90 \\
\hline
\end{tabular}

En général, le groupe 2 obtient les moyennes les plus favorables pour les deux types de contributions, personnelles et gouvernementales. Quant au groupe 1, il montre des moyennes plus élevées pour les contributions personnelles que pour les contributions gouvernementales; au contraire, le groupe 3 se montre plus positif à l'égard des contributions gouvernementales tout en se révélant le moins favorable à l'égard des contributions personnelles.

Pour le principe organisateur des contributions personnelles, les groupes 1 et 2 sont plus positifs à l'égard des droits élémentaires que le groupe 3 . Dans les trois autres classes de droits, le groupe 2 est le plus positif, suivi du groupe 1 ; le groupe 3 se montre le moins positif, principalement en ce qui trait aux droits civils et politiques. Pour le principe organisateur des contributions gouvernementales, le groupe 2 se démarque des autres par ses moyennes les plus élevées dans chaque classe de droits. Les trois groupes sont favorables aux droits élémentaires, mais le groupe 1 est le moins positif. Pour les droits socioéconomiques, la moyenne du groupe 2 est la plus élevée de toutes les moyennes obtenues. Pour les droits civils-politiques, le groupe 2 est également le plus positif des trois, suivi du groupe 3 ; le groupe 1 est le moins positif. Enfin, pour l'ordre 
social, les groupes 1 et 3 ont des moyennes similaires, inférieures à celle du groupe 2 .

De ces résultats, trois tendances se dessinent: a) une tendance favorable aux contributions individuelles et institutionnelles du groupe 2 , jugé optimiste à l'égard des DH; b) une tendance positive à l'égard des contributions personnelles et sceptique face aux contributions institutionnelles du groupe 1, qualifié de personnaliste et c) une tendance défavorable à l'égard des contributions personnelles et optimiste par rapport aux contributions gouvernementales du groupe 3, qualifié de gouvernementaliste. La distribution des sujets $(n=286)$ par faculté (psychologie, droit, sciences et travail social) selon ces trois profils de réponses n'indique aucune différence significative.

4. L'ancrage des variations individuelles sur la structure des valeurs, la perception des tensions sociales et des injustices personnelles

Les valeurs

Une analyse en composantes principales a été effectuée sur une échelle réduite de 31 à 21 items. Cette dernière a été construite à partir des 18 items de l'échelle de Rokeach, de deux items de Bond et d'un indice global calculé à partir de la moyenne des scores obtenus sur les 11 items tirés du Préambule de la charte des $\mathrm{DH}$, dont la consistance interne s'était révélée satisfaisante (alpha de Cronbach =0,93).

Cinq facteurs, expliquant $59 \%$ du total de la variance, ont été retenus; le tableau 5 en montre la saturation et la communauté. Les cinq types de valeurs qui se dégagent sont: a) des valeurs fondamentales (facteur $1: 30 \%$ de variance expliquée); ce sont des valeurs dites universelles (paix, égalité, liberté, etc.); b) des valeurs personnelles (facteur $2: 9 \%$ de variance expliquée), telles que l'amitié véritable, la sagesse, l'harmonie intérieure et avec les autres, etc.; c) des valeurs familiales (facteur $3: 7 \%$ de variance expliquée), comme la sécurité familiale, le confort, le bonheur, une vie accomplie; d) des valeurs sociales (facteur 4: 7\% de variance expliquée), telles qu'une vie excitante, l'accomplissement amoureux, la reconnaissance sociale, voire la sécurité nationale; e) des valeurs traditionnelles (facteur 5: 6\% de variance expliquée) : le salut et le respect des traditions. 
Tableau 5

Résultats de l'analyse factorielle sur les échelles de valeurs

\begin{tabular}{|c|c|c|c|c|c|c|}
\hline \multirow[b]{2}{*}{ Échelles } & \multicolumn{4}{|c|}{$\begin{array}{l}\text { Saturations } \\
\text { Facteurs }\end{array}$} & \multirow[b]{2}{*}{5} & \multirow[b]{2}{*}{ Commun. } \\
\hline & 1 & 2 & 3 & 4 & & \\
\hline Monde en paix & 0,80 & 0,15 & 0,18 & 0,06 & 0,11 & 0,70 \\
\hline Préambule & 0,79 & 0,33 & 0,00 & 0,09 & 0,19 & 0,77 \\
\hline Égalité & 0,78 & 0,20 & 0,07 & 0,00 & 0,10 & 0,66 \\
\hline Liberté & 0,65 & 0,07 & 0,13 & 0,22 & $-0,16$ & 0,51 \\
\hline Monde en beauté & 0,52 & 0,02 & 0,03 & 0,33 & 0,13 & 0,40 \\
\hline Amitié & 0,13 & 0,71 & 0,16 & 0,18 & $-0,14$ & 0,60 \\
\hline Harmonie avec autres & 0,26 & 0,67 & 0,23 & 0,18 & 0,16 & 0,63 \\
\hline Harmonie intérieure & 0,07 & 0,65 & 0,37 & $-0,03$ & 0,17 & 0,59 \\
\hline Sagesse & 0,22 & 0,63 & $-0,36$ & 0,04 & 0,23 & 0,62 \\
\hline Respect de soi & 0,29 & 0,48 & 0,41 & 0,24 & $-0,07$ & 0,54 \\
\hline Vie confortable & $-0,12$ & $-0,06$ & 0,68 & 0,38 & 0,10 & 0,64 \\
\hline Bonheur & 0,13 & 0,35 & 0,60 & 0,20 & 0,09 & 0,55 \\
\hline Sécurité familiale & 0,48 & 0,14 & 0,58 & 0,01 & 0,11 & 0,60 \\
\hline Vie accomplie & 0,34 & 0,23 & 0,56 & 0,11 & $-0,02$ & 0,49 \\
\hline Plaisir & 0,15 & 0,08 & 0,18 & 0,76 & 0,12 & 0,65 \\
\hline Vie excitante & 0,22 & 0,17 & $-0,04$ & 0,67 & $-0,36$ & 0,65 \\
\hline Amour & 0,10 & 0,22 & 0,29 & 0,55 & 0,14 & 0,47 \\
\hline Reconnaissance sociale & 0,01 & 0,12 & 0,31 & 0,47 & 0,36 & 0,46 \\
\hline Sécurité nationale & 0,42 & 0,00 & 0,14 & 0,44 & 0,36 & 0,51 \\
\hline Salut & 0,11 & $-0,01$ & 0,12 & $-0,01$ & 0,81 & 0,68 \\
\hline Respect des traditions & 0,16 & 0,34 & $-0,06$ & 0,23 & 0,60 & 0,56 \\
\hline Variance expliquée & 0,30 & 0,09 & 0,07 & 0,07 & 0,06 & \\
\hline
\end{tabular}

Note: Saturations et communauté après rotation varimax.

L'analyse factorielle des tensions

Une analyse factorielle a été réalisée sur les résultats de l'échelle de perception de tensions et conflits par rapport aux différentes catégories de groupes sociaux. Trois facteurs expliquant $61 \%$ de la variance ont été obtenus. Le premier facteur (41\% de variance expliquée) regroupe les tensions et conflits qui touchent les caractéristiques individuelles et interpersonnelles, tels l'âge, le sexe, la langue, les origines familiales, etc.; on le nomme conflits interpersonnels. Le deuxième facteur, appelé conflits 
ethniques ( $11 \%$ de variance expliquée), regroupe les tensions qui touchent l'origine ethnique, telles la race, la couleur de la peau et la nationalité. Le troisième facteur $(9 \%$ de la variance expliquée) est constitué des tensions provoquées par les diverses opinions, convictions et religions. Ce facteur est nommé conflits idéologiques (voir tableau 6).

\section{Tableau 6}

Analyse factorielle sur les perceptions des tensions et conflits par rapport aux différentes catégories de groupes sociaux

Saturations

Tensions et conflits entre individus selon :

Facteur 1 Facteur 2 Facteur 3 Commun.

\begin{tabular}{lrrrr}
\hline Âge différent & $\mathbf{0 , 7 6}$ & 0,04 & 0,05 & 0,59 \\
Origine familiale différente & $\mathbf{0 , 7 3}$ & 0,11 & 0,25 & 0,61 \\
Origine sociale différente & $\mathbf{0 , 6 9}$ & 0,26 & 0,34 & 0,66 \\
Vivant dans région différente & $\mathbf{0 , 6 7}$ & 0,22 & 0,15 & 0,51 \\
Fortune différente & $\mathbf{0 , 6 5}$ & 0,07 & 0,26 & 0,49 \\
Langue différente & $\mathbf{0 , 5 3}$ & 0,42 & $-0,10$ & 0,47 \\
Sexe différent & $\mathbf{0 , 5 0}$ & 0,46 & 0,17 & 0,50 \\
Race différente & 0,10 & $\mathbf{0 , 8 9}$ & 0,20 & 0,85 \\
Couleur de peau différente & 0,14 & $\mathbf{0 , 8 9}$ & 0,16 & 0,83 \\
Nationalité différente & 0,36 & $\mathbf{0 , 5 7}$ & 0,34 & 0,56 \\
Opinion différente & 0,27 & 0,13 & $\mathbf{0 , 7 6}$ & 0,67 \\
Conviction différente & 0,23 & 0,07 & $\mathbf{0 , 7 5}$ & 0,62 \\
Religion différente & 0,04 & 0,34 & $\mathbf{0 , 7 4}$ & 0,66 \\
Variance expliquée & 0,41 & 0,11 & 0,09 &
\end{tabular}

Total de variance expliquée: $61 \%$

Note: Saturations et communauté après rotation varimax.

L'analyse factorielle de la perception des injustices personnelles

L'analyse factorielle effectuée sur la perception d'injustices personnelles montre quatre facteurs (61\% de variance expliquée). Le premier facteur ( $29 \%$ de la variance expliquée), injustices idéologiques et culturelles, regroupe les injustices personnelles perçues en regard des opinions, des convictions, de la religion, de la langue et de la région dans laquelle l'individu vit. Le deuxième facteur ( $13 \%$ de la variance expliquée), injustices socio-économiques, regroupe l'origine familiale, la fortune 
personnelle et l'origine sociale. Le troisième facteur (10\% de la variance expliquée), injustices ethniques, se rapporte à la race, à la couleur de la peau et à la nationalité. Enfin, le quatrième facteur ( $9 \%$ de la variance expliquée), injustices physiques, renvoie au sexe et à l'âge (voir tableau 7).

Tableau 7

Analyse factorielle sur les perceptions d'injustices personnelles à l'égard des différentes catégories de groupes sociaux

Perception d'injustices Saturations

Facteur 1 Facteur 2 Facteur 3 Facteur 4 Commun.

\begin{tabular}{lrrrrr}
\hline Opinions personnelles & $\mathbf{0 , 8 0}$ & 0,10 & 0,12 & $-0,14$ & 0,68 \\
Convictions personnelles & $\mathbf{0 , 7 0}$ & 0,31 & 0,05 & 0,01 & 0,59 \\
Région où l'on vit & $\mathbf{0 , 5 9}$ & 0,16 & $-0,20$ & 0,14 & 0,44 \\
Langue qu'on parle & $\mathbf{0 , 5 7}$ & $-0,08$ & 0,24 & 0,36 & 0,52 \\
Religion & $\mathbf{0 , 4 9}$ & 0,15 & 0,08 & 0,07 & 0,27 \\
Fortune dont on dispose & 0,20 & $\mathbf{0 , 7 8}$ & $-0,03$ & $-0,04$ & 0,66 \\
Origine familiale & 0,13 & $\mathbf{0 , 7 7}$ & 0,24 & 0,15 & 0,69 \\
Origine sociale & 0,23 & $\mathbf{0 , 7 6}$ & 0,20 & 0,24 & 0,72 \\
Race & 0,07 & 0,09 & $\mathbf{0 , 8 8}$ & $-0,03$ & 0,79 \\
Couleur de peau & $-0,02$ & 0,15 & $\mathbf{0 , 8 8}$ & $-0,01$ & 0,79 \\
Nationalité & 0,45 & 0,15 & $\mathbf{0 , 5 1}$ & 0,22 & 0,53 \\
Sexe & $-0,07$ & 0,07 & 0,09 & $\mathbf{0 , 8 7}$ & 0,77 \\
Âge & 0,26 & 0,22 & $-0,12$ & $\mathbf{0 , 5 3}$ & 0,41 \\
Variance expliquée & 0,29 & 0,13 & 0,09 & 0,08 & \\
Total de variance expliquée: $: 61 \%$ & & & & \\
\hline
\end{tabular}

Note : Saturations et communauté après rotation varimax.

Une analyse discriminante sur les trois groupes de répondants (optimiste, personnaliste et gouvernementaliste), effectuée selon la procédure Wilks (stepwise) ${ }^{8}$, permet d'observer l'ancrage des deux principes organisateurs (contributions personnelles et contributions gouvernementales) dégagés du champ des RSDH, dans la structure factorielle des valeurs, de la perception des tensions à l'égard de diverses catégories sociales et des injustices personnelles s'y rapportant. Deux fonctions se révèlent significatives, la fonction $1\left(\chi^{2}(14)=53,48 ; \mathrm{p}<0,001\right)$ qui explique $77,8 \%$ de la variance et la deuxième fonction $\left(\chi^{2}(6)=12,581\right.$; $\mathrm{p}<0,05)$, qui explique $22,2 \%$ de la variance. Les corrélations entre les variables discriminantes et les fonctions canoniques 
indiquent que la fonction 1 est centrée de façon positive sur les valeurs fondamentales et personnelles et que la fonction 2 est centrée a) de façon positive sur les injustices physiques et les conflits idéologiques; et b) de façon négative sur les injustices socio-économiques et ethniques (voir tableau 8).

Tableau 8

Corrélations entre variables et fonctions canoniques discriminantes

\begin{tabular}{lrr}
\hline & \multicolumn{2}{c}{ Corrélations } \\
Variables discriminantes & Fonction $\mathbf{1}$ & Fonction 2 \\
\hline Valeurs fondamentales & $\mathbf{0 , 7 9}$ & 0,12 \\
Valeurs personnelles & $\mathbf{0 , 3 6}$ & $-0,09$ \\
Valeurs familiales & $-0,21$ & 0,19 \\
Injustices socio-économiques & 0,04 & $-\mathbf{0 , 6 2}$ \\
Injustices ethniques & 0,02 & $-\mathbf{0 , 4 8}$ \\
Injustices physiques & $-0,04$ & $\mathbf{0 , 4 4}$ \\
Conflits idéologiques & $-0,22$ & $\mathbf{0 , 4 3}$ \\
Injustices culturelles & $-0,02$ & 0,17 \\
Conflits ethniques & 0,15 & 0,15 \\
Valeurs traditionnelles & 0,07 & $-0,13$ \\
Valeurs sociales & 0,04 & $-0,08$ \\
Conflits interpersonnels & $-0,02$ & 0,06 \\
\hline
\end{tabular}

Note: En gras, corrélations $\geq 0,30$.

Les deux fonctions permettent de classer ${ }^{9} 52 \%\left(\chi^{2}(4)=37,34\right.$; $\mathrm{p}<0,001)$ du total des sujets. Pour le groupe 1 , la prédiction de classement est de $36,4 \%\left(\chi^{2}(1)=6,73 ; p<0,001\right)$, pour le groupe 2 , elle est de $67,2 \%\left(\chi^{2}(1)=13,34 ; \mathrm{p}<0,001\right)$ et pour le groupe 3 , de $44,4 \%$ $\left(\chi^{2}(1)=15,97 ; p<0,001\right)$. La première fonction permet donc de distinguer les individus qui montrent un attachement aux valeurs fondamentales et personnelles. La deuxième fonction permet de distinguer ceux qui accordent de l'importance aux injustices physiques et socio-économiques et aux conflits idéologiques et ethniques par rapport à ceux qui ne s'en soucient pas. Les centroïdes des groupes indiquent que, dans la fonction 1, le groupe 2 (optimiste) s'oppose nettement au groupe 3 (gouvernementaliste), le groupe 1 (personnaliste) se situant dans une position intermédiaire. La fonction 2 distingue le groupe 1 (personnaliste) des groupes 2 (optimiste) et 3 (gouvernementaliste) qui se montrent très proches sur cette fonction (voir tableau 9). 
Tableau 9

Centroïdes des fonctions discriminantes selon les groupes de sujets

\begin{tabular}{lrrr}
\hline Groupes de sujets & & Fonction 1 & Fonction 2 \\
\hline 1. Personnaliste & $(\mathrm{n}=68)$ & $-0,02$ & $-0,51$ \\
2. Optimiste & $(\mathrm{n}=146)$ & 0,41 & 0,13 \\
3. Gouvernementaliste & $(\mathrm{n}=72)$ & $-0,74$ & 0,14 \\
\hline
\end{tabular}

Le groupe 1 (personnaliste), plus positif à l'égard des contributions personnelles que gouvernementales, est celui qui accorde le plus d'importance aux injustices socio-économiques et ethniques; il donne une importance mitigée aux valeurs fondamentales et personnelles. Le groupe 2 (optimiste), qui se montre le plus favorable à l'égard des contributions individuelles et gouvernementales, est celui qui accorde le plus d'importance aux valeurs fondamentales et personnelles. Par ailleurs, il montre une certaine sensibilité aux injustices physiques et aux conflits idéologiques et, à un moindre degré, aux injustices socio-économiques et ethniques. Le groupe 3 (gouvernementaliste), plus positif à l'égard des contributions gouvernementales, est celui qui se montre le plus détaché des valeurs fondamentales et personnelles. En outre, ce groupe, tout comme le deuxième, montre une certaine sensibilité aux injustices physiques et aux conflits idéologiques et moins à l'égard des injustices socio-économiques et ethniques.

\section{DISCUSSION ET CONCLUSION}

À l'issue de ce long travail d'analyse, la présente étude a permis une nouvelle fois de mettre en évidence, à travers les réponses des sujets, une classification des droits de l'hommes similaire à celle proposée par René Cassin. Ces résultats attestent la présence d'un savoir commun des DH chez la population visée par l'enquête, appuyant la thèse que les notions des DH sont devenues des référents universels. En effet, quatre classes de droits ont pu être dégagées: la première regroupe des droits élémentaires; dans la deuxième se retrouvent des droits civils et politiques; la troisième se rapporte à des droits socio-économiques et la quatrième, à des droits d'ordre social. 
L'analyse des structures sous-jacentes à cette classification révèle l'existence de trois dimensions qui se résument de la façon suivante: la première, qui affiche une polarisation entre droits fondamentaux et devoirs à l'égard de la communauté, montre le caractère lié des deux types de notions constitutives de la DUDH, à savoir les droits et les devoirs, et rend significatives les maintes controverses qu'ils ont suscitées tout au long de l'évolution historique des droits de l'homme (voir Gauchet, 1989). Cette polarisation illustre bien comment tout droit comporte des limites qui s'actualisent à travers des créances exigées par la communauté. Dans le cas particulier de nos sujets, ces limites sont associées aux notions de démocratie, de bien-être et d'ordre social.

La deuxième dimension montre une opposition entre, d'une part, le droit de libre circulation et de certains droits socioéconomiques (travail, liberté d'opinion, mariage) et, d'autre part, le droit d'asile, certains droits civils et politiques (reconnaissance juridique) et d'ordre social (ordre public et international). Cette dimension illustre un double dilemme: celui qui exige que la jouissance d'un droit soit accompagnée de règles qui en assurent l'application sans aucune discrimination et celui qui réclame que tout bénéfice d'un droit rencontre sa limite dans le respect des droits des autres : "tout ce qui est mon droit est aussi le droit d'autrui, de sorte que je ne puis réclamer pour mon compte sans vouloir garantir chez les autres» (Gauchet, 1989: 80). La mise en évidence de ce principe dans la population étudiée est d'autant plus significative que le Québec, province qui par tradition connaît un flux migratoire régulier, fait face, de manière constante, au dilemme du respect des droits à la libre circulation et à l'asile par rapport aux droits et aux besoins des Québécois eux-mêmes.

La troisième dimension met en opposition les droits qui touchent la sphère individuelle et ceux qui touchent la sphère communautaire. Cette dimension illustre les exigences liées aux deux grands modèles de société : celui qui favorise principalement les libertés civiles et politiques des individus et celui qui privilégie plutôt les droits socio-économiques et culturels de la communauté (voir Vasak, 1990). Cette opposition n'est en rien surprenante ici puisque la société québécoise, dans laquelle vivent nos sujets, puise dans ces deux modèles; d'une part, sa politique institutionnelle est celle d'un État-providence (bienêtre social, assurance-maladie, garantie de l'éducation pour tous); d'autre part, le gouvernement s'est doté d'une fonction 
régulatrice pour assurer le respect des garanties et des droits individuels (ouverture des marchés, libre concurrence, libre entreprise).

La population ciblée, composée d'étudiants inscrits dans six champs disciplinaires, montre différents profils par rapport à ces trois dimensions. Les groupes de science politique-sociologie, sciences et collège utilisent de façon semblable et modérée ces trois principes; la dimension des droits fondamentaux et de responsabilité à l'égard de la communauté y est toutefois privilégiée. Cela nous porte à croire que ces groupes d'étudiants ont une conception étendue des droits de l'homme. Cette vision élargie chez trois groupes hétérogènes peut s'expliquer de la façon suivante: dans le cas de science politique-sociologie, elle peut résulter d'une réflexion obligée par le choix disciplinaire; dans le cas des collégiens et des étudiants en sciences, elle est peut-être la manifestation d'une connaissance générale mais vague du domaine, attribuable à l'âge ou à des orientations disciplinaires autres.

Les autres groupes ont des préoccupations plus différenciées. Chez le groupe de travail social, la dimension libre circulation versus asile est privilégiée à toute autre et de façon marquée. Pour le groupe de psychologie, cette même dimension est utilisée, mais de façon plus modérée et équivalant à celle de la sphère individuelle versus la sphère communautaire. Enfin, le groupe de droit utilise surtout cette dernière dimension puis, de façon plus modérée, la dimension de droit fondamental versus responsabilité à l'égard de la communauté (droit / devoir). La lecture de ces résultats semble montrer que ces trois profils reflètent partiellement des préoccupations liées aux champs disciplinaires respectifs. Dans le cas du groupe de travail social, le choix serait lié à des problèmes rencontrés dans l'exercice de la profession, par exemple les problèmes d'immigration, la famille ; pour le groupe de psychologie, ce même choix, accompagné de celui qui est lié à l'individu par rapport à la communauté, rejoint aussi des préoccupations professionnelles. Enfin, pour le groupe de droit, les intérêts disciplinaires amènent les sujets à se projeter dans la dimension droit / devoir et dans celle qui touche les droits spécifiques liés au statut civil et juridique de l'individu et ceux qui se préoccupent des rapports socio-économiques de la communauté.

Par ailleurs, les deux principes organisateurs déjà mis en évidence dans d'autres travaux (Doise et Herrera, 1994; Doise, Spini, Jesuino, Ng et Emler, 1994), à savoir: les contributions 
personnelles et les contributions institutionnelles par rapport aux $\mathrm{DH}$, se retrouvent également dans la présente étude. À l'égard de ces deux principes, trois profils d'individus se dessinent. Un premier profil, qu'on pourrait qualifier de personnaliste, se montre sceptique à l'égard des contributions institutionnelles et croit plutôt aux contributions personnelles; un deuxième profil, optimiste, considère de façon positive les deux types de contributions; enfin, un troisième profil, gouvernementaliste, croit surtout aux contributions gouvernementales.

Ces profils se distinguent par l'importance accordée aux types de valeurs, de perceptions de conflits et d'injustices personnelles à l'égard de différentes catégories sociales. Le premier profil se différencie par son ancrage dans les injustices socioéconomiques et les conflits ethniques. Le deuxième, au contraire, est ancré dans les valeurs fondamentales (dignité, liberté, égalité) et personnelles (harmonie interne, estime de soi, sagesse, etc.). Le troisième profil est celui qui affiche la plus grande opposition à l'égard des valeurs fondamentales et personnelles.

De l'ensemble de ces observations, il est possible d'inférer que le profil du groupe 1 (personnaliste) se rapproche d'une conception libérale à l'égard des applications des droits de l'homme. Ses croyances, plutôt centrées sur les contributions personnelles, le rendent très sensible aux injustices socio-économiques et ethniques. Chez ce groupe, les normes $(\mathrm{DH})$ sont vues comme étant applicables au groupe à travers l'individu, principalement en ce qui a trait aux droits socio-économiques (p. ex.: droit à la propriété privée, droit au travail). Cette vision se rapproche de la notion de droit naturel chez l'homme: tout individu, de par sa nature, a des droits dès les premiers moments de son existence. La gestion des DH devient ainsi d'abord la responsabilité de l'individu en fonction de ses intérêts et objectifs personnels, laissant au second plan les rôles institutionnels (voir Gauchet, 1989; Madiot, 1991). Pour les optimistes (profil 2), les DH semblent être des droits communs à l'homme social; selon ce point de vue, tant les institutions que les individus ont un rôle actif à jouer. Alors que les premières doivent assurer la régulation des échanges interindividuels, les seconds doivent se montrer responsables dans leurs échanges symboliques de valeurs (Gauchet, 1989). Enfin, on peut dire des gouvernementalistes (profil 3) que leur vision des DH est fonctionnelle. D'un côté, ce groupe dote l'État d'un rôle instrumental dans la gestion des DH; c'est l'État qui est responsable des mécanismes sociaux 
qui règlent et sanctionnent les rapports entre les membres de la société. D'un autre côté, il se montre opposé aux valeurs fondamentales et personnelles. On peut donc supposer que, chez ce groupe, la notion des DH a perdu son statut de «normes»; ils ne deviennent alors qu'un ensemble de règles sociales, dissociées des valeurs, des codes et des intérêts individuels.

En conclusion, cette étude sur les représentations sociales des droits de l'homme (RSDH) a permis de mettre en évidence certains aspects universels des DH et des différences interindividuelles chez une population d'étudiants francophones québécois. Cette première étude en milieu québécois a également fait ressortir certaines caractéristiques particulières. À travers les réponses des individus concernant les RSDH, il a été possible de distinguer des profils de prises de position à l'égard de ces droits et leurs ancrages dans les valeurs, tensions et injustices ressenties dans la vie quotidienne des individus. Les résultats ont également révélé certains des éléments dynamiques qui soustendent les échanges symboliques de valeurs des personnes interrogées. Ces valeurs, parfois partagées, parfois antagonistes, au sein d'une même population, se sont manifestées avec plus ou moins d'importance selon les intérêts, attentes et expériences des individus concernés.

Dans cette société pluriethnique qu'est le Québec, il a été intéressant de constater, au vu du climat politique actuel, où la population est tiraillée par des visions antagonistes du modèle de société désiré, combien les enjeux sont multiples et complexes. Il n'est donc pas surprenant que différents profils d'individus, parfois semblables, parfois opposés, aient émergé des résultats. Ils ne font que révéler une nouvelle fois à quel point les projets que l'on nourrit pour un idéal de société sont tributaires des croyances, intérêts et valeurs des individus qui la composent. De tels résultats peuvent dès maintenant servir de cadre de référence, en milieu québécois, pour saisir certaines nuances contenues dans les discours populaires sur les DH et ainsi mieux comprendre les enjeux qui y sont rattachés.

En outre, nos résultats montrent la richesse méthodologique qu'offre le champ d'étude des RSDH pour cerner l'univers symbolique des membres de toute société, ici celle des Québécois, et les dynamiques mises en place pour permettre aux divers codes, normes et valeurs propres à chaque culture de s'harmoniser à l'intérieur du code de justice international que constitue la Déclaration universelle des droits de l'homme. 


\section{Notes}

1. Plusieurs personnes ont rendu cette recherche possible. Nous tenons à remercier plus spécialement les professeurs R. Bourhis et A. Jacob de l'Université du Québec à Montréal, M. Deniger de l'Université de Montréal, R. Auclair, L. Balthazar, R. Caillibot, J. Caillouette, D. Delâge, M. Dupras, C. Ferron, A. Fortin, D. Fortin, J. Himmelman, P. Issalys, B. Melkevik, P. Morin, M. Olivier et G. Otis de l'Université Laval, de même que les responsables du programme de Sciences humaines du collège François-Xavier-Garneau qui, en nous autorisant à intervenir auprès des étudiants de leurs cours, ont permis la récolte des données de cette étude.

Pour de plus amples renseignements sur la recherche, communiquez avec le Laboratoire de psychologie interculturelle, École de psychologie, Université Laval, Québec, Canada, G1K 7P4.

2. La Déclaration universelle des droits de l'homme, établie par les 58 États qui constituaient alors l'Organisation des Nations unies, contient les normes générales du système juridique global, c'est-à-dire au niveau de l'homme. Les buts poursuivis par cette charte sont de promouvoir le bien-être de tout être humain et de satisfaire ses besoins essentiels en améliorant les conditions socioéconomiques qui favorisent son développement et son épanouissement et en assurant une protection pour maintenir ces conditions dans le plus grand respect des uns et des autres. Les 30 articles de cette déclaration énoncent les droits reconnus de tout être humain, à savoir : les articles 1 et 2 stipulent que« tous les êtres humains naissent libres et égaux en dignité et en droits », les articles 3 à 21 énoncent les droits civils et politiques (le droit à la vie, la liberté de pensée, etc.), les articles 22 et 27 énoncent les droits économiques, sociaux et culturels (le droit à la sécurité sociale, le droit au travail, etc.) et les articles 28 à 30 reconnaissent à toute personne le droit à un ordre social et international et ils soulignent les devoirs et responsabilités de l'individu envers la communauté (Nations unies, 1990).

3. René Cassin (voir Agi, 1980) classe les droits de la DUDH de la façon suivante: les droits des principes fondamentaux (articles 1 et 2), les droits de la personne (articles 3 à 11), les droits des rapports entre les personnes (articles 12 à 17), les droits des libertés publiques (articles 18 à 21), les droits économiques et sociaux (articles 22 à 27) et, enfin, les droits relatifs à l'ordre international (articles 28 à 30).

4. L'Université du Québec à Montréal et l'Université de Montréal.

5. En raison du très faible nombre d'étudiants en sociologie $(n=11)$ qui ont participé à cette étude, leurs réponses ont été regroupées avec celles des étudiants en science politique.

6. La formule mathématique du cœefficient de dissimilitude est la suivante: $\operatorname{dij}=\left[(\mathrm{Ai}-\mathrm{Aj}) 2+(\mathrm{Bi}-\mathrm{Bj}) 2+(\mathrm{Ci}-\mathrm{Cj})^{2}+(\mathrm{Di}-\mathrm{Dj})^{2}+(\mathrm{Ei}-\mathrm{Ej})^{2}+(\mathrm{Fi}-\mathrm{Fj})^{2}+(\mathrm{Gi}-\mathrm{G} \mathbf{j})^{2}+(\mathrm{Hi}-\mathrm{Hj})^{2}\right]^{1 / 2}$ $\mathrm{i}$ et $\mathrm{j}=$ numéros des articles ( 1 à 30$)$; $\mathrm{A}$ à $\mathrm{H}=$ chacune des huit échelles considérées (supra).

7. Cette procédure a été retenue en raison de l'indice de consistance interne élevée obtenu sur les huit échelles (alpha de Cronbach entre 0,92 à 0,97).

8. Cette procédure est retenue parce qu'elle prend en considération à la fois les différences et l'homogénéité inter-groupes. Étant donné que le Wilks lambda est un indicateur statistique inverse, le stepwise Wilks lambda sélectionne la variable qui produit le plus petit lambda à chaque étape (W. Klecka, Discriminant Analysis, 1980, p. 54).

9. Cette classification a été validée selon la méthode «cross-validation », effectuée sur la proportion de $70 \%$ et $30 \%$ de la population. 


\section{Références bibliographiques}

AGI, M. (1980). De l'idée d'universalité comme fondatrice du concept des droits de l'Homme: d'après la vie et l'oeuvre de René Cassin. Antibes: Éditions Alp'Azur.

BERCIS, P. (1993). Guide des droits de l'homme: la conquête des libertés. Paris : Éditions Hachette Éducation.

BOND, M.H. (1988). «Finding universal dimensions of individual variation in multicultural studies of values: The Rokeach and Chinese value surveys», Journal of Personality and Social Psychology, vol. 55, $\mathrm{n}^{\circ} 2: 143-164$.

Boudon, R. (1977). Effets pervers et ordre social. Paris : Presses Universitaires de France.

Boudon, R. (1995). Le juste et le vrai. Étude sur l'objectivité des valeurs et de la connaissance. Paris: Éditions Fayard.

Bourdieu, P. (1991). «Les Juristes, Gardiens de l'Hypocrisie Collective», dans F. Chazel et J. Commaille (dir.), Normes juridiques et régulation sociale. Paris : Librairie Générale de Droit et de Jurisprudence.

ClÉmenCE, A. et W. DOISE (1995). «La représentation sociale de la justice: Une approche des droits dans la pensée ordinaire », Année Sociologique.

ClémenCe, A., W. Doise et F. LOREnZI-CiOldi (1994). «Prises de position et principes organisateurs des représentations sociales », dans C. Guimelli (dir.), Structures et transformations des représentations sociales (p. 119-152). Neuchâtel et Paris: Delachaux et Niestlé.

Clémence, A., W. Doise, A.M. De Rosa et L. GonZalez (1995). « La représentation sociale des droits de l'homme: Une recherche internationale sur l'étendue et les limites de l'universalité », Journal International de Psychologie, vol. 30 : 181-212.

CôTÉ, A. et L. LEMONDE (1988). Discrimination et Commission des droits de la personne. Montréal : Éditions Saint-Martin.

Delmas-Marty, M., W. Doise, R. Lenoir et A. PerCheron (1989). «La perception des droits de l'homme dans la société contemporaine: Projet d'enquête à partir de la jurisprudence de la Cour européenne des droits de l'homme », Archives de Politique Criminelle, $\mathrm{n}^{\circ} 11: 35-58$.

DoIse, W. (1991/1992). «L'ancrage dans les études sur les représentations sociales», Bulletin de Psychologie, $\mathrm{n}^{\circ} 45$ : 189-195.

Doise, W., P. Dell'Ambrogio et D. SPINi (1991). "Psychologie sociale et droits de l'homme ", Revue internationale de psychologie sociale, $\mathrm{n}^{\circ} 4: 257-277$.

Doise, W., A. ClÉmenCE et F. LORENZI-CiOldi (1992). Représentations sociales et analyses de données. Grenoble: Presses Universitaires de Grenoble.

DOISE, W. et M. HERRERA (1994). «Déclaration universelle et représentations sociales des droits de l'homme: une étude à Genève», Revue internationale de psychologie sociale, 7, 85-105. 
DoISE, W., D. SPINI, J.C. Jesuino, S.H. NG et N. EMLER (1994). «Values and perceived conflicts in the social representations of human rights : feasibility of a cross-national study», Swiss Journal of Psychology, $\mathrm{n}^{\circ} 53$ : 240-251.

GAUCHET, M. (1989). La révolution des droits de l'homme. Paris: Gallimard.

GAY, D. (1985). "Réflexions critiques sur les politiques ethniques du gouvernement fédéral canadien 1971-1985 et du gouvernement du Québec », Revue internationale d'action communautaire, 14/54.

HAIR, J., R. ANDERSON, R. TATHAM et W. BLACK (1987). Multivariate Data Analysis. Toronto: Maxwell Macmillan Canada.

KLECKA, W. (1980). Discriminant Analysis. Beverly Hills : Sage Publications.

MAdiot, Y. (1991). Droits de l'Homme. Paris: Masson.

Moscovici, S. (1976). La psychanalyse, son image et son public (2e édition). Paris: Presses Universitaires de France.

NATIONS UNIES (1994). Rapport mondial sur le développement humain. Programme des Nations Unies pour le développement (PNUD). New York: Economica.

NG, S.H., A.B.M. AKhtar Hossain, P. Ball, M.H. Bond, K. Hayashi, S.P. LIM, M.P. O'DRISCOLL, D. SiNHA et K.S. YANG (1982). «Human Values in nine countries» dans R. Rath, H.S. Asthana, D. Sinha et J.B.H. Sinha (dir.). Diversity and Unity in Cross-Cultural Psychology. Liesse : Swets and Zeitlinger B.V.

PASSERON, J.C. (1991). Le raisonnement sociologique. L'espace non poppérien du raisonnement naturel. Paris : Éditions Nathan.

PEDHAZUR, E.J. (1982). Multiple regression in behavioral research (2 édition). Fort Worth, Texas : Holt, Rinehart et Winston.

ROCHER, F. et G. ROCHER (1991). «La culture québécoise en devenir : les défis du pluralisme» dans F. Ouellet et M. Pagé (dir.), Pluriethnicité, éducation et société. Construire un espace commun (p. 43-76). Québec: Institut québécois de recherche sur la culture.

RokeACH, M. (1973). The Nature of Human Values. New York and London: The Free Press.

VASAK, K. (1990). "Les différentes catégories des droits de 1'Homme", dans A. Lapeyre, F. De Tinguy et K. Vasak (dir.), Les dimensions universelles des droits de l'Homme. Vol. 1. Unesco: Bruxelles.

YAlDen, M.F. (1988). Pour une promotion concertée de l'égalité dans l'emploi (Notes pour une allocution). Ottawa: Commission canadienne des droits de la personne. 